\title{
Phase 1 study of pembrolizumab (MK-3475; anti-PD-1 monoclonal antibody) in Japanese patients with advanced solid tumors
}

\author{
Toshio Shimizu ${ }^{1}$ - Takashi Seto ${ }^{2} \cdot$ Fumihiko Hirai $^{2} \cdot$ Mitsuhiro Takenoyama $^{2}$. \\ Kaname Nosaki $^{2}$. Junji Tsurutani ${ }^{1}$. Hiroyasu Kaneda ${ }^{1}$ - Tsutomu Iwasa ${ }^{1}$. \\ Hisato Kawakami $^{1} \cdot$ Kazuo Noguchi $^{3}$ - Takashi Shimamoto ${ }^{3} \cdot$ Kazuhiko Nakagawa $^{1}$
}

Received: 9 March 2016 / Accepted: 16 March 2016/Published online: 22 March 2016

(C) The Author(s) 2016. This article is published with open access at Springerlink.com

Summary Background This phase I study evaluated the safety and tolerability, pharmacokinetics and pharmacodynamics, immunogenicity, and antitumor activity of pembrolizumab in Japanese patients with advanced solid tumors. Methods Following an initial dose and a 28-day rest (cycle 1), pembrolizumab was administered as an intravenous infusion at escalating doses ( 2 or $10 \mathrm{mg} / \mathrm{kg}$ ) every 2 weeks (Q2W) until disease progression or unacceptable toxicity. Adverse events (AEs) were assessed using CTCAE v4.0, and tumor response was assessed using both RECIST v1.1 and immune-related response criteria (irRC). Full pharmacokinetic sampling was performed during cycle 1. Results Three patients received pembrolizumab at $2.0 \mathrm{mg} / \mathrm{kg}$ and seven at $10 \mathrm{mg} / \mathrm{kg}$. No dose-limiting toxicities were observed during cycle 1. Eighty percent of patients experienced drug-related AEs (mostly grade 1 or 2); the most common drug-related AEs were nausea, malaise, pyrexia, and aspartate aminotransferase/alanine transaminase (AST/ALT) elevations $(n=2$ each). No drugrelated grade 4 or 5 AEs occurred. Immune-related AEs comprised grade 3 ALT elevation $(n=1)$, grade 3 AST elevation $(n=1)$, grade 1 pneumonitis $(n=1)$, and grade 1 thyroidstimulating hormone elevation $(n=1)$. The safety and

Toshio Shimizu

jcog9511@hotmail.co.jp

1 Department of Medical Oncology, Kindai University Faculty of Medicine, 377-2 Ohno-higashi, Osaka-sayama, Osaka 589-8511, Japan

2 Department of Thoracic Oncology, National Kyushu Cancer Center, 3-1-1 Notame, Minami-ku, Fukuoka 811-1395, Japan

3 MSD K.K., Kitanomaru square, 1-13-12 Kudan-kita, Chiyoda-ku, Tokyo 102-8667, Japan pharmacokinetic profiles of Japanese patients were similar to those previously reported for Caucasian patients. A partial tumor response was observed in one patient with non-smallcell lung cancer (NSCLC) and in one patient with melanoma. Conclusions Pembrolizumab at both 2 and $10 \mathrm{mg} / \mathrm{kg}$ Q2W was well tolerated in Japanese patients with advanced solid tumors and showed encouraging anti-tumor activity against melanoma and NSCLC.

Keywords Pembrolizumab - Anti-PD-1 therapy · Pharmacokinetics $\cdot$ Phase I study $\cdot$ PD-L1

\section{Introduction}

The PD-1 pathway represents a major immune control switch that may be engaged by tumor cells to overcome active T-cell immune surveillance [1]. The ligands for PD-1 (PD-L1 and PD-L2) are constitutively expressed or can be induced in various tumors, including melanoma [2-5]. The high expression of PD-L1 (and to a lesser extent, PD-L2) on tumor cells is correlated with poor prognosis and poor survival in various cancer types, including renal cell carcinoma, pancreatic carcinoma, hepatocellular carcinoma, ovarian carcinoma, and nonsmall-cell lung cancer (NSCLC) [6-10]. Furthermore, it has been suggested that PD-1 regulates tumor-specific T-cell expansion in patients with melanoma [11]. Preclinical in vitro and in vivo experiments have shown that PD-1 and/or PD-L1 blockade using monoclonal antibodies enhances tumor cellspecific T-cell activation, cytokine production, anti-tumor effector mechanisms, and the clearance of tumor cells by the immune system [12-16]. PD-1 and PD-L1 inhibitors have validated PD-1 as an attractive target for clinical therapeutic intervention. PD-1 inhibition was tested in a clinical study of 
patients with a range of solid tumor types, and promising clinical activity was noted in multiple tumor types, including melanoma and NSCLC [17].

Pembrolizumab (formerly known as MK-3475) is a potent, highly selective, IgG4-k humanized monoclonal antibody that prevents PD-1 from binding with PD-L1 and PD-L2. This agent was generated by grafting the variable region sequences of a mouse antihuman PD-1 antibody onto a human IgG4-k isotype framework containing a stabilizing S228P mutation of the Fc region. Pembrolizumab exhibited high affinity for the PD-1 receptor, strong inhibition of PD-L1 and PD-L2, and robust activity in a functional ex vivo T-cell modulation assay using human donor blood cells (data on file; Merck \& Co., Inc.).

A first-in-human phase 1 study was conducted to evaluate the safety, pharmacokinetics, and pharmacodynamics of pembrolizumab in non-Japanese patients with advanced solid tumors. No dose-limiting toxicities (DLTs) were observed, and the maximum administered dose (MAD) was $10 \mathrm{mg} / \mathrm{kg}$ every 2 weeks (Q2W). Pharmacokinetic and pharmacodynamic analyses showed that the lowest dose with the full potential for antitumor activity was $2 \mathrm{mg} / \mathrm{kg}$ every 3 weeks (Q3W) [18].

In the present study, the safety and tolerability, pharmacokinetics (PK), and immunogenicity of pembrolizumab were investigated in Japanese patients with advanced solid tumors. The tumor response to pembrolizumab was also evaluated as an exploratory objective.

\section{Materials and methods}

\section{Patient eligibility}

This study was conducted based on the Declaration of Helsinki and the Guidelines for the Clinical Evaluation Methods of Anti-Cancer Drugs in Japan (Japanese Ministry of Health, Labour, and Welfare notification, November 1, 2005). The study was approved by the institutional review board of each study site.

The main eligibility criteria were as follows: a histologically or cytologically confirmed diagnosis of locally advanced or metastatic solid tumors in a patient who had experienced disease progression while on standard therapy or in a patient intolerant of, or not eligible for standard therapy; a patient age of 20 years or older; an Eastern Cooperative Oncology Group (ECOG) performance status of 0 or 1 ; and adequate hematologic, hepatic, and renal functions. The exclusion criteria included the administration of chemotherapy, radiotherapy, or biological therapy in the 4 weeks ( 2 weeks for palliative radiotherapy and kinase inhibitors) prior to enrollment; previous treatment with a PD-1, PD-L1, or cytotoxic Tlymphocyte-associated protein 4 inhibitor; untreated and/or unstable central nervous system metastasis; or the presence of autoimmune disease.

All patients provided informed consent, and the study was conducted in accordance with current Good Clinical Practice standards. This study was registered at ClinicalTrials.gov as NCT01840579.

\section{Study design and evaluation}

This study was an open-label, non-randomized, phase 1 study of pembrolizumab in Japanese patients with advanced solid tumors that was conducted at two sites in Japan. This study was designed to investigate the safety and tolerability, PK, immunogenicity, and anti-tumor activity of pembrolizumab monotherapy. Pembrolizumab was administered as a $30-\mathrm{min}$ intravenous (i.v.) infusion at a dose of 2 or $10 \mathrm{mg} / \mathrm{kg}$. Dose escalation was conducted using the conventional " $3+3$ design," with cohorts of three patients sequentially enrolled at pembrolizumab doses of 2 and $10 \mathrm{mg} / \mathrm{kg}$ administered on days 1 and 28 and Q2W thereafter until disease progression or intolerable toxicity occurred. The initial 28 days after the first administration (cycle 1) were regarded as the DLT evaluation period. Three or six patients were enrolled at each dose based on the toxicity probability intervals [19]. In the DLT assessments, if none of the three patients or none/one of the six patients had a DLT at a certain dose, that dose was considered to be tolerable.

Adverse events (AEs) were graded using the National Cancer Institute Common Terminology Criteria for AEs, version 4.0. A DLT was defined as any of the following events occurring during cycle 1 (the initial 28 days): grade 4 neutropenia lasting for $\geq 7$ days; grade 3 or 4 neutropenia with a fever $>38.5^{\circ} \mathrm{C}$ and/or infection requiring antibiotic or anti-fungal treatment; grade 4 thrombocytopenia; grade 4 nonhematologic toxicity; or a grade 3 non-hematologic toxicity (laboratory value) persisting for $>1$ week or requiring medical intervention.

Anti-tumor activity was evaluated at baseline and every 6 weeks according to the Response Evaluation Criteria In Solid Tumors, version 1.1 (RECIST v1.1) and the immunerelated response criteria (irRC) [20].

\section{Pharmacokinetics and immunogenicity (presence of anti-drug antibody)}

Blood samples for the PK analyses were collected predose, postdose ( $<30 \mathrm{~min}$ after infusion), and 6,24 , and $48 \mathrm{~h}$ after the start of the first infusion; on days 8, 15, 22, and 29 of cycle 1 ; predose and postdose in cycle 2 and every other cycle thereafter for the first 12 months; and 30 days after the last pembrolizumab dose. The pembrolizumab serum concentrations were quantified using a validated electrochemiluminescent assay (lower limit of quantification, $10 \mathrm{ng} / \mathrm{mL}$ ). The PK data are 
described using a noncompartmental approach. Blood samples for anti-drug antibody (ADA) analyses were collected predose and $24 \mathrm{~h}$ after the start of the first infusion, predose in cycle 2 and every other cycle thereafter for the first 12 months, and 30 days after the last pembrolizumab dose.

The presence of ADA was determined using bridging electrochemiluminescence and was evaluated using the standard three-step method consisting of a screening test, a confirmation test, and an antibody titer test. ADA was determined to be positive if the result for at least one predose or postdose sample was positive on the confirmation test.

\section{PD-L1 expression}

PD-L1 expression was measured using immunohistochemistry performed on formalin-fixed, paraffin-embedded tissue sections at QualTek Clinical Laboratories. PD-L1 positivity was defined as staining in $\geq 1 \%$ of the tumor cells (modified proportion score) or stroma using immunohistochemistry with the PD-L1 22C3 antibody.

\section{Results}

\section{Patient characteristics}

Ten Japanese patients with advanced solid tumors were enrolled and were evaluated in this study; three patients were treated with $2 \mathrm{mg} / \mathrm{kg}$ Q2W and seven patients were treated with $10 \mathrm{mg} / \mathrm{kg} \mathrm{Q} 2 \mathrm{~W}$. The baseline characteristics of the patients are summarized in Table 1. The age range was 52.091.0 years (median: 62.0 years), and the most frequent solid tumors were NSCLC $(5 / 10,50.0 \%)$ and melanoma $(3 / 10$, $30.0 \%$ ). The median number of prior chemotherapy regimens was 5 (range, $0-13$ ). The median number of treatments was 4 (range, 1-18). At the time of data cutoff, nine of the ten patients had discontinued pembrolizumab treatment: one because of an adverse event (grade 4 cerebral infarction that was considered by the investigator to be unrelated to pembrolizumab treatment) and eight because of disease progression.

\section{Safety and tolerability}

The DLTs occurring during cycle 1 were evaluated. One patient treated with pembrolizumab $10 \mathrm{mg} / \mathrm{kg}$ was excluded from the DLT evaluation because the patient discontinued the study because of disease progression during the DLT evaluation period. Therefore, that patient was replaced with a new patient for the DLT evaluation. No DLTs were observed in the three patients treated with $2 \mathrm{mg} / \mathrm{kg}$ or in the six patients treated with $10 \mathrm{mg} / \mathrm{kg}$. The drug-related AEs reported for all treatment cycles at both dose levels are summarized in Table 2.
Table 1 Baseline patient characteristics

\begin{tabular}{ll}
\hline Characteristic & Value \\
\hline Number of patients & 10 \\
Age, year & \\
Median & 62.0 \\
Range & $52.0-91.0$ \\
Sex & \\
Male & 5 \\
Female & 5 \\
Weight, kg & \\
Median & 61.7 \\
Range & $39.4-79.9$ \\
ECOG performance status & \\
0 & 4 \\
1 & 6 \\
Primary tumor & \\
NSCLC & 5 \\
Melanoma & 3 \\
Breast cancer & 1 \\
Extramammary Paget's disease & 1 \\
Number of prior systemic therapies & \\
Median & $5-13$ \\
Range &
\end{tabular}

ECOG Eastern Cooperative Oncology Group

Eighty percent of patients experienced drug-related AEs (mostly grade 1 or 2 ). The most common AEs related to pembrolizumab treatment were nausea, malaise, pyrexia, elevated alanine transaminase (ALT) levels, and elevated aspartate aminotransferase (AST) levels (2/10 [20\%] for each AE). No drug-related grade 4 or grade 5 AEs occurred in this study. None of the patients discontinued pembrolizumab treatment because of a drug-related AE. One patient (a 53-year-old man) with advanced NSCLC developed grade 3 ALT elevation, grade 3 AST elevation, and grade 1 pneumonitis (onset, day 42 for each); after the discontinuation of pembrolizumab treatment, he developed grade 3 hyponatremia (onset, day 56). The grade 3 ALT/AST elevations resolved after treatment with glycyrrhetinic acid, and the grade 3 hyponatremia resolved with sodium chloride supplement. Overall, one case each of grade 3 ALT elevation, grade 3 AST elevation, grade 1 pneumonitis, and grade 1 thyroid-stimulating hormone (TSH) elevation were reported as immune-related AEs by the investigators.

\section{Pharmacokinetic and immunogenicity evaluation}

The mean serum concentration profiles for pembrolizumab are shown in Fig. 1, and descriptive statistics for the PK parameters are given in Table 3 and Fig. 2. The serum concentration of pembrolizumab following the first i.v. administration 
Table 2 All grades of adverse events related to pembrolizumab treatment
Fig. 1 Serum concentration-time profiles of pembrolizumab following the first administration. Symbols and error bars indicate the mean \pm SD

\begin{tabular}{|c|c|c|c|c|c|c|}
\hline & \multicolumn{2}{|c|}{$\begin{array}{l}2 \mathrm{mg} / \mathrm{kg}, \mathrm{Q} 2 \mathrm{~W} \\
(n=3)\end{array}$} & \multicolumn{2}{|c|}{$\begin{array}{l}10 \mathrm{mg} / \mathrm{kg}, \mathrm{Q} 2 \mathrm{~W} \\
(n=7)\end{array}$} & \multicolumn{2}{|l|}{$\begin{array}{l}\text { Total } \\
(n=10)\end{array}$} \\
\hline & All Grades & Grade 3 & All Grades & Grade 3 & All Grades & Grade 3 \\
\hline \multicolumn{7}{|c|}{ Blood and lymphatic system disorders } \\
\hline Anemia & 0 & 0 & 1 & 0 & 1 & 0 \\
\hline Neutropenia & 0 & 0 & 1 & 0 & 1 & 0 \\
\hline Thrombocytopenia & 0 & 0 & 1 & 0 & 1 & 0 \\
\hline \multicolumn{7}{|l|}{ Gastrointestinal disorders } \\
\hline Nausea & 1 & 1 & 1 & 0 & 2 & 0 \\
\hline \multicolumn{7}{|c|}{ General disorders and administration site conditions } \\
\hline Malaise & 1 & 0 & 1 & 0 & 2 & 0 \\
\hline Pyrexia & 1 & 0 & 1 & 0 & 2 & 0 \\
\hline Fatigue & 1 & 0 & 0 & 0 & 1 & 0 \\
\hline \multicolumn{7}{|l|}{ Infections and infestations } \\
\hline Infection & 0 & 0 & 1 & 0 & 1 & 0 \\
\hline \multicolumn{7}{|l|}{ Investigations } \\
\hline ALT elevation & 0 & 0 & 2 & 1 & 2 & 1 \\
\hline AST elevation & 0 & 0 & 2 & 1 & 2 & 1 \\
\hline Blood cholesterol elevation & 1 & 0 & 0 & 0 & 1 & 0 \\
\hline Blood TSH elevation & 0 & 0 & 1 & 0 & 1 & 0 \\
\hline Weight reduction & 0 & 0 & 1 & 0 & 1 & 0 \\
\hline \multicolumn{7}{|c|}{ Metabolism and nutrition disorders } \\
\hline Dehydration & 0 & 0 & 1 & 0 & 1 & 0 \\
\hline Hyperglycemia & 0 & 0 & 1 & 0 & 1 & 0 \\
\hline Hyperkalemia & 0 & 0 & 1 & 0 & 1 & 0 \\
\hline Hypertriglyceridemia & 1 & 0 & 0 & 0 & 1 & 0 \\
\hline Hypokalemia & 0 & 0 & 1 & 0 & 1 & 0 \\
\hline Hyponatremia & 0 & 0 & 1 & 1 & 1 & 1 \\
\hline \multicolumn{7}{|c|}{ Respiratory, thoracic, and mediastinal disorders } \\
\hline Exertional dyspnea & 0 & 0 & 1 & 0 & 1 & 0 \\
\hline Pneumonitis & 0 & 0 & 1 & 0 & 1 & 0 \\
\hline \multicolumn{7}{|c|}{ Skin and subcutaneous tissue disorders } \\
\hline Drug eruption & 0 & 0 & 1 & 0 & 1 & 0 \\
\hline Rash & 0 & 0 & 1 & 0 & 1 & 0 \\
\hline Urticaria & 0 & 0 & 1 & 0 & 1 & 0 \\
\hline
\end{tabular}

AEs related to pembrolizumab were grade 1, 2, or 3 in severity, with no grade 4 or 5 AEs reported

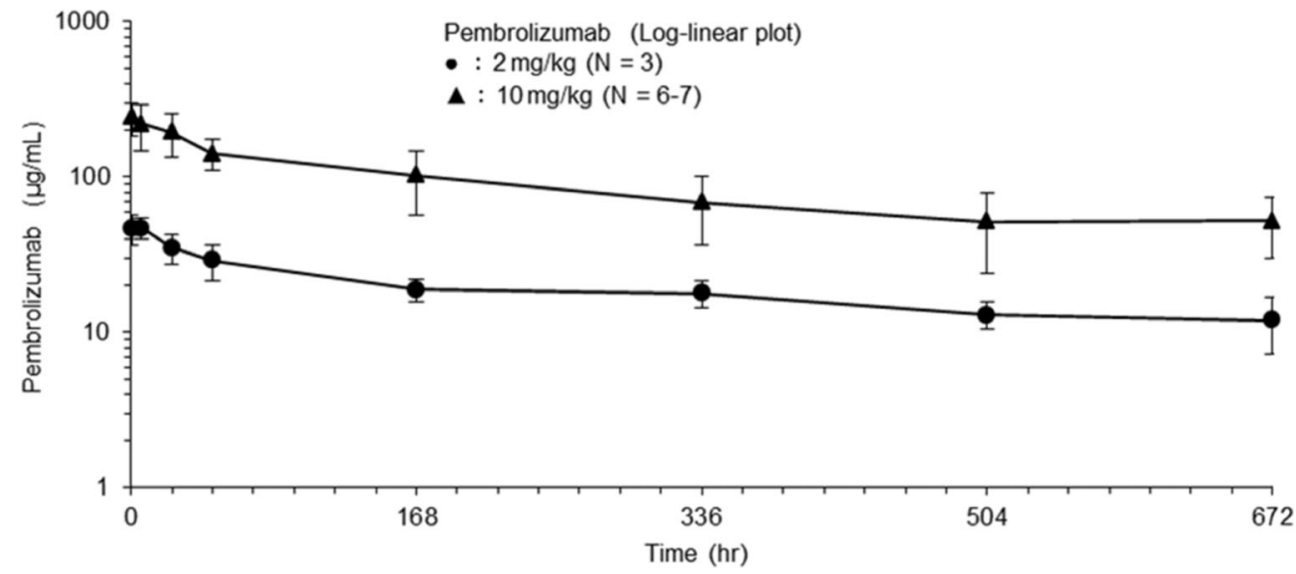


Table 3 Summary of pharmacokinetic parameters following the first dose (2 or $10 \mathrm{mg} / \mathrm{kg}$ ) of pembrolizumab in Japanese patients with advanced solid tumors

\begin{tabular}{|c|c|c|c|c|c|c|c|}
\hline Dose & $\begin{array}{l}\mathrm{C}_{\max ,} \mu \mathrm{g} / \mathrm{mL}, \\
\mathrm{GM}(\mathrm{CV} \%)\end{array}$ & $\begin{array}{l}\mathrm{T}_{\max , \text { days, }} \\
\text { median (range) }\end{array}$ & $\begin{array}{l}\mathrm{AUC}_{0-28}, \mu \mathrm{g} \text { day } / \mathrm{mL} \\
\mathrm{GM}(\mathrm{CV} \%)\end{array}$ & $\begin{array}{l}\mathrm{AUC}_{0-\infty}, \mu \mathrm{g} \text { day } / \mathrm{mL}, \\
\mathrm{GM}(\mathrm{CV} \%)\end{array}$ & $\begin{array}{l}\mathrm{t}_{1 / 2} \text {, days, } \\
\mathrm{GM}(\mathrm{CV} \%)\end{array}$ & $\begin{array}{l}\mathrm{Vz}, \mathrm{mL} / \mathrm{kg} \\
\mathrm{GM}(\mathrm{CV} \%)\end{array}$ & $\begin{array}{l}\text { CL, mL/day/kg, } \\
\text { GM (CV\%) }\end{array}$ \\
\hline $\begin{array}{l}2 \mathrm{mg} / \mathrm{kg} \\
(n=3)\end{array}$ & $\begin{array}{l}47.4 \\
(19)\end{array}$ & $\begin{array}{l}0.22 \\
(0.002-0.23)\end{array}$ & $\begin{array}{l}507 \\
(20)\end{array}$ & $\begin{array}{l}812 \\
(45)\end{array}$ & $\begin{array}{l}18.4 \\
(56)\end{array}$ & $\begin{array}{l}65.3 \\
(21)\end{array}$ & $\begin{array}{l}2.46 \\
(45)\end{array}$ \\
\hline $\begin{array}{l}10 \mathrm{mg} / \mathrm{kg} \\
(n=7)\end{array}$ & $\begin{array}{l}250 \\
(23)\end{array}$ & $\begin{array}{l}0.01 \\
(0.001-0.23)\end{array}$ & $\begin{array}{l}2219 \\
(32)\end{array}$ & $\begin{array}{l}3410 \\
(56)\end{array}$ & $\begin{array}{l}18.1 \\
(68)\end{array}$ & $\begin{array}{l}76.5 \\
(34)\end{array}$ & $\begin{array}{l}2.93 \\
(56)\end{array}$ \\
\hline
\end{tabular}

$C_{\max }$ maximum observed serum concentration, $T_{\max }$ time of maximum observed serum concentration, $A U C_{0-28}$ area under the concentration-time curve from day 0 to day $28, A U C_{0-\infty}$ area under the concentration-time curve from day 0 to infinity, $t_{1 / 2}$ elimination half-life, $V z$ the terminal phase volume, $C L$ clearance, $G M$ geometric mean, $C V$ coefficient of variation

decreased slowly in Japanese patients with advanced solid tumors. The geometric mean terminal half-lives $\left(t_{1 / 2}\right)$ for pembrolizumab following the first i.v. administration were 18.4 and 18.1 days at doses of 2 and $10 \mathrm{mg} / \mathrm{kg}$, respectively, in Japanese patients. For doses of 2 and $10 \mathrm{mg} / \mathrm{kg}$, respectively, the geometric mean (and coefficient of variation) values for the clearance (CL) were 2.46 (45\%) and 2.93 (56\%) mL/day/kg and for the terminal phase volume (Vz) were $65.3(21 \%)$ and $76.5(34 \%) \mathrm{mL} / \mathrm{kg}$. The $\mathrm{t}_{1 / 2}, \mathrm{CL}$, and $\mathrm{Vz}$ values were very similar for doses of 2 and $10 \mathrm{mg} / \mathrm{kg}$ in Japanese patients with advanced solid tumors. Exposure to pembrolizumab, as reflected by the total area under the concentration-time curve $\left(A U C_{0-\infty}\right)$ and maximum concentration $\left(\mathrm{C}_{\max }\right)$, following the first i.v. administration of pembrolizumab at doses of 2 and $10 \mathrm{mg} / \mathrm{kg}$ generally increased in a dose-proportional manner in Japanese patients. The PK profiles of pembrolizumab, which has a low clearance, a limited volume of distribution, and a long $t_{1 / 2}$, are typical of therapeutic antibodies. The trough concentration increased during repeated dosing at 2 or $10 \mathrm{mg}$ / $\mathrm{kg}$ Q2W. Based on the $\mathrm{t}_{1 / 2}$ (18 days) and the dose interval (2 weeks), approximately 3.5 months (cycle 8 ) were considered necessary to achieve a steady state. However, only two patients treated with $2 \mathrm{mg} / \mathrm{kg}$ and one patient treated with $10 \mathrm{mg} / \mathrm{kg}$ were still receiving pembrolizumab at cycle 8 . Considering the limited PK data available for doses of 2 and $10 \mathrm{mg} / \mathrm{kg}$ beyond cycle 8 , the steady state trough concentration could not be determined based on the observed PK data.

ADA was not detected at any time in screening assays from predose through to cycle 18 (day 140), except in a predose assay performed during cycle 1 in one patient;
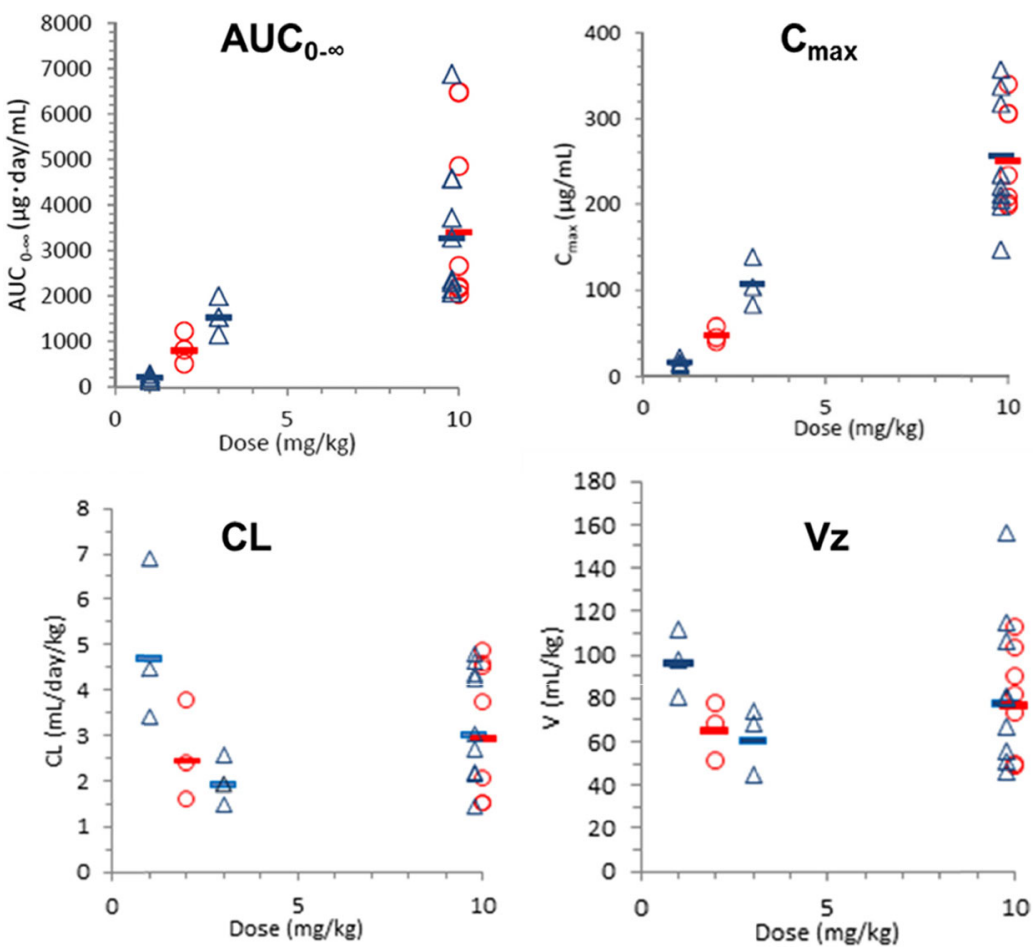

- Non-JPN mean ( $n=3$ to 10$)$

$\triangle$ Non-JPN individual

JPN mean $(n=3$ to 7$)$

- JPN individual

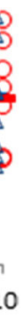

Fig. 2 PK parameters versus dose following the first administration of pembrolizumab in Japanese and non-Japanese patients with advanced $C_{\max }$ maximum concentration, $C L$ clearance, $V z$ the terminal phase volume 
however, the results of the confirmatory assay were negative for this patient.

\section{Antitumor activity and PD-L1 expression}

As an exploratory objective, the tumor response to pembrolizumab was evaluated according to the RECIST v1.1 and irRC criteria. One patient with extramammary Paget's disease was excluded from the tumor response assessment because the patient did not have any measurable lesions at the study baseline. Among the nine patients who were evaluated, partial responses (as determined by an investigator review according to RECIST v1.1) were observed in two patients $(22.2 \%)$ treated with pembrolizumab $10 \mathrm{mg} / \mathrm{kg}$ Q2W; one patient (a 91-year-old man) had metastatic melanoma (time to response: 46 days), and the other (a 53-year-old man) had NSCLC (time to response: 41 days). The two patients also had partial responses as determined by an investigator review according to irRC. The patient with advanced NSCLC developed immune-related AEs (grade 3 ALT/AST elevations and grade 1 pneumonitis) at the same time as the observation of the partial response. At the time of data cutoff, the patient with advanced metastatic melanoma who had achieved a partial response was continuing to receive pembrolizumab treatment because, despite disease progression according to RECIST v1.1, disease progression was not evident according to irRC (response duration: more than 225 days) (Fig. 3). Tumor tissue from five patients was available to undergo a PD-L1 expression immunohistochemistry assay. PD-L1 expression was positive in tumor samples from two patients and negative in tumor samples from three patients. No responses were observed in the two patients with PD-L1-positive tumors by an investigator review whereas a

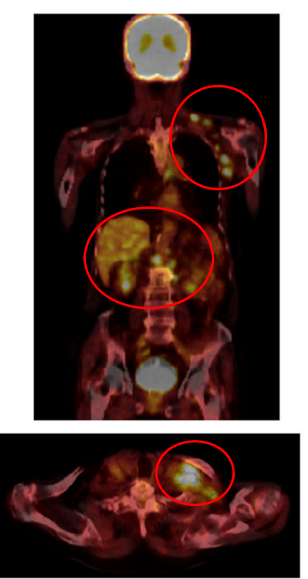

Baseline: 1 Aug 2013

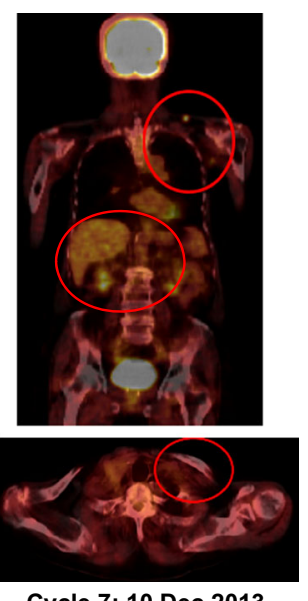

Cycle 7: 10 Dec 2013
Fig. 3 PET-CT images taken before the start of treatment and after cycle 7 show evidence of antitumor activity. A rapid and durable partial response according to irRC was observed in a 91-year-old man with advanced metastatic acral lentiginous melanoma who had active disease in the liver and multiple lymph nodes when treatment with pembrolizumab at $10 \mathrm{mg} / \mathrm{kg}$ Q2W was initiated partial response was observed in one of the three patients with PD-L1-negative tumors.

\section{Discussion}

The primary objective of the present study was to investigate the safety and tolerability of single-agent pembrolizumab administered in Japanese patients with advanced solid tumors. The dosing schedules in the present study were selected based on the results of a phase 1 study of pembrolizumab in nonJapanese patients with advanced solid tumors. The previous study showed that the MAD was $10 \mathrm{mg} / \mathrm{kg}$ Q2W, and the lowest dose with the full potential for antitumor activity was $2 \mathrm{mg} / \mathrm{kg}$ Q3W [18]. No DLTs were observed at either dose in the present study. The most common drug-related AEs were nausea, malaise, pyrexia, and AST/ALT elevations $(n=2$ each). One case each of grade 3 ALT elevation, grade 3 AST elevation, grade 1 pneumonitis, and grade 1 TSH elevation were reported as immune-related AEs. The AEs observed in the present study have also been reported for clinical studies of pembrolizumab in non-Japanese patients. Although the number of Japanese patients was limited, the safety profile of pembrolizumab in Japanese patients with advanced solid tumors in the present study was generally consistent with that observed previously in non-Japanese patients. Drug-induced autoimmune-like toxicities have been observed in patients treated with immune checkpoint inhibitors (such as those used in anti-PD-1 therapy) through the infiltration of immune cells into normal noncancerous tissues. Immune-related AEs can affect multiple organs such as the skin, bowel, kidney, peripheral and central nervous system, liver, lymph nodes, eyes, pancreas, and endocrine tissues. Steroid therapy is recommended for the management of immune-related AEs [21, 22]. In the present study, most AEs were of grade 1 or 2 and were manageable by interrupting pembrolizumab treatment and/or medical intervention without steroid therapy.

Pembrolizumab exposure at a dose of $10 \mathrm{mg} / \mathrm{kg}$ was generally similar for Japanese and non-Japanese patients with advanced solid tumors $\left(\mathrm{AUC}_{0-\infty}: 3410\right.$ vs. $3270 \mu \mathrm{g}$ day $/ \mathrm{mL}, \mathrm{C}_{\max }: 250$ vs. $256 \mu \mathrm{g} / \mathrm{mL}$, respectively). The mean $\mathrm{CL}, \mathrm{t}_{1 / 2}$, and $\mathrm{Vz}$ values were generally similar between the two populations for doses in the range 1$10 \mathrm{mg} / \mathrm{kg}$. In addition, the individual values for $\mathrm{AUC}_{0-\infty}$, $\mathrm{C}_{\max }, \mathrm{t}_{1 / 2}, \mathrm{Vz}$, and $\mathrm{CL}$ for doses in the range $1-10 \mathrm{mg} / \mathrm{kg}$ overlapped (Fig. 2) [18]. These results indicated that the PK of pembrolizumab in Japanese patients with advanced solid tumors was generally similar to that in non-Japanese patients with advanced solid tumors.

As an exploratory analysis, the tumor responses were also evaluated. Partial responses were observed in two of the nine patients $(22.2 \%)$ treated with $10 \mathrm{mg} / \mathrm{kg}$ Q2W by an 
investigator review according to both RECIST v1.1 and irRC criteria. One of the five patients with advanced NSCLC and one of the three patients with advanced melanoma achieved partial responses. A durable response (response duration of more than 225 days according to irRC) was observed in one patient with metastatic acral lentiginous melanoma, which is the most common subtype of melanoma in Asian countries (superficial spreading melanoma is more common in Caucasians). This result is encouraging for the further development of pembrolizumab treatment for melanoma in Japan.

Because of the limited number of patients and tumor samples, a relationship between tumor response and PD-L1 expression was not observed in the present study. Recent data have shown that PD-L1 expression, microsatellite instability high, increased mutation burden, and the immune gene expression signature are correlated with the improved efficacy of pembrolizumab [23-26], indicating the importance of biomarker development to identify patients suitable for anti-PD-1 and anti-PD-L1 therapy. Based on the safety data from this phase 1 study, Japanese patients are currently being enrolled in late-phase global studies of pembrolizumab for the treatment of various types of tumors, such as NSCLC, gastric cancer, head and neck cancer, bladder cancer, and colorectal cancer. Further biomarker analyses in these clinical studies are on-going.

Recent randomized studies of pembrolizumab demonstrated that no clinically meaningful differences in the safety and efficacy of pembrolizumab were evident between $2 \mathrm{mg} / \mathrm{kg}$ Q3W and $10 \mathrm{mg} / \mathrm{kg}$ Q3W or between $10 \mathrm{mg} / \mathrm{kg}$ Q3W and $10 \mathrm{mg} / \mathrm{kg}$ Q2W [27-31]. The administration of pembrolizumab at $2 \mathrm{mg} / \mathrm{kg}$ Q3W is currently approved in the United States and other countries for the treatment of patients with unresectable or metastatic melanoma or metastatic NSCLC expressing PD-L1.

In conclusion, pembrolizumab at dosages of 2 and $10 \mathrm{mg} /$ $\mathrm{kg}$ Q2W was well tolerated in Japanese patients with advanced solid tumors and showed encouraging anti-tumor activity against melanoma and NSCLC.

Acknowledgments The authors would like to thank Mikio Ishii (MSD K.K.) for the PK analysis and Shi Rong Han (MSD K.K.) for the statistical analysis. Research funding was provided by MSD K.K. (Tokyo, Japan).

\section{Compliance with ethical standards}

Disclosure statement Takashi Shimamoto and Kazuo Noguchi are employees of MSD K.K. and hold stock and stock options in Merck \& Co., Inc. The remaining authors have no potential conflicts of interest to report.

Ethical standards The study was performed in accordance with the ethical standards laid down in the 1964 Declaration of Helsinki and its later amendments. The study was approved by the institutional review board of each study site, and all the patients provided informed consent prior to their inclusion in the study.
Open Access This article is distributed under the terms of the Creative Commons Attribution 4.0 International License (http:// creativecommons.org/licenses/by/4.0/), which permits unrestricted use, distribution, and reproduction in any medium, provided you give appropriate credit to the original author(s) and the source, provide a link to the Creative Commons license, and indicate if changes were made.

\section{References}

1. Disis ML (2010) Immune regulation of cancer. J Clin Oncol 28: $4531-4538$

2. Dong H, Strome SE, Salomao DR, Tamura H, Hirano F, Flies DB et al (2002) Tumor-associated B7-H1 promotes T-cell apoptosis: a potential mechanism of immune evasion. Nat Med 8:793-800

3. Sharpe AH, Freeman GJ (2002) The B7-CD28 superfamily. Nat Rev Immunol 2:116-126

4. Brown JA, Dorfman DM, Ma F-R, Sullivan EL, Munoz O, Wood $C R$ et al (2003) Blockade of programmed death-1 ligands on dendritic cells enhances $\mathrm{T}$ cell activation and cytokine production. $\mathrm{J}$ Immunol 170:1257-1266

5. Francisco LM, Sage PT, Sharpe AH (2010) The PD-1 pathway in tolerance and autoimmunity. Immunol Rev 236:219-242

6. Thompson RH, Dong H, Lohse CM, Leibovich BC, Blute ML, Cheville JC et al (2007) PD-1 is expressed by tumor-infiltrating immune cells and is associated with poor outcome for patients with renal cell carcinoma. Clin Cancer Res 13:1757-1761

7. Nomi T, Sho M, Akahori T, Hamada K, Kubo A, Kanehiro H et al (2007) Clinical significance and therapeutic potential of the programmed death-1 ligand/programmed death-1 pathway in human pancreatic cancer. Clin Cancer Res 13:2151-2157

8. Gao Q, Wang X-Y, Qiu S-J, Yamato I, Sho M, Nakajima Y et al (2009) Overexpression of PD-L1 significantly associates with tumor aggressiveness and postoperative recurrence in human hepatocellular carcinoma. Clin Cancer Res 15:971-979

9. Hamanishi J, Mandai M, Iwasaki M, Okazaki T, Tanaka Y, Yamaguchi K et al (2007) Programmed cell death 1 ligand 1 and tumor-infiltrating CD8+ T lymphocytes are prognostic factors of human ovarian cancer. Proc Natl Acad Sci U S A 104:3360-3365

10. Mu CY, Huang JA, Chen Y, Chen C, Zhang XG (2011) High expression of $\mathrm{PD}-\mathrm{L} 1$ in lung cancer may contribute to poor prognosis and tumor cells immune escape through suppressing tumor infiltrating dendritic cells maturation. Med Oncol 28:682-688

11. Fourcade J, Kudela P, Sun Z, Shen H, Land SR, Lenzner D et al (2009) PD-1 is a regulator of NY-ESO-1-specific CD8+ T cell expansion in melanoma patients. J Immunol 182:5240-5249

12. Gao Q, Wang XY, Qiu SJ, Yamato I, Sho M, Nakajima Y et al (2009) Overexpression of PD-L1 significantly associates with tumor aggressiveness and postoperative recurrence in human hepatocellular carcinoma. Clin Cancer Res 15:971-979

13. Blank C, Mackensen A (2007) Contribution of the PD-L1/PD-1 pathway to T-cell exhaustion: an update on implications for chronic infections and tumor evasion. Cancer Immunol Immunother 56: 739-745

14. Tsushima F, Tanaka K, Otsuki N, Youngnak P, Iwai H, Omura K et al (2006) Predominant expression of B7-H1 and its immunoregulatory roles in oral squamous cell carcinoma. Oral Oncol 42:268274

15. Iwai Y, Ishida M, Tanaka Y, Okazaki T, Honjo T, Minato N (2002) Involvement of PD-L1 on tumor cells in the escape from host immune system and tumor immunotherapy by PD-L1 blockade. Proc Natl Acad Sci U S A 99:12293-12297

16. Sznol M, Powderly JD, Smith DC, Brahmer JR, Drake CG, McDermott DF et al (2010) Safety and antitumor activity of 
biweekly MDX-1106 (anti-PD-1, BMS-936558/ONO-4538) in patients with advanced refractory malignancies [Abstract]. J Clin Oncol 28(Suppl):2506

17. Brahmer JR, Drake CG, Wollner I, Powderly JD, Picus J, Sharfman WH et al (2010) Phase I study of single-agent anti-programmed death-1 (MDX-1106) in refractory solid tumors: safety, clinical activity, pharmacodynamics, and immunologic correlates. J Clin Oncol 28:3167-3175

18. Patnaik A, Kang SP, Rasco D, Papadopoulos KP, Elassaiss-Schaap J, Beeram M et al (2015) Phase I study of pembrolizumab (MK3475; anti-PD-1 monoclonal antibody) in patients with advanced solid tumors. Clin Cancer Res 21:4286-4293

19. Ji Y, Li Y, Nebiyou Bekele B (2007) Dose-finding in phase I clinical trials based on toxicity probability intervals. Clin Trials 4:235-244

20. Wolchok JD, Hoos A, O'Day S, Weber JS, Hamid O, Lebbé C et al (2009) Guidelines for the evaluation of immune therapy activity in solid tumors: immune-related response criteria. Clin Cancer Res 15: 7412-7420

21. Chen TW, Razak AR, Bedard PL, Siu LL, Hansen AR (2015) A systematic review of immune-related adverse event reporting in clinical trials of immune checkpoint inhibitors. Ann Oncol 26: $1824-1829$

22. Weber JS, Kähler KC, Hauschild A (2012) Management of immune-related adverse events and kinetics of response with ipilimumab. J Clin Oncol 30:2691-2697

23. Garon EB, Rizvi NA, Hui R, Leighl N, Balmanoukian AS, Eder JP et al (2015) Pembrolizumab for the treatment of non-small-cell lung cancer. N Engl J Med 372:2018-2028

24. Le DT, Uram JN, Wang H, Bartlett BR, Kemberling H, Eyring AD et al (2015) PD-1 Blockade in tumors with mismatch-repair deficiency. N Engl J Med 372:2509-2520
25. Rizvi NA, Hellmann MD, Snyder A, Kvistborg P, Makarov V, Havel JJ et al (2015) Cancer immunology. Mutational landscape determines sensitivity to PD-1 blockade in non-small cell lung cancer. Science 348:124-128

26. Ribas A, Robert C, Hodi FS, Wolchok JD, Joshua AM, Hwu WJ et al. (2015) Association of response to programmed death receptor 1 (PD-1) blockade with pembrolizumab (MK-3475) with an interferon-inflammatory immune gene signature [Meeting Abstract]. J Clin Oncol 33 (suppl; abstr 3001)

27. Ribas A, Puzanov I, Dummer R, Schadendorf D, Hamid O, Robert $\mathrm{C}$ et al (2015) Pembrolizumab versus investigatorchoice chemotherapy for ipilimumab-refractory melanoma (KEYNOTE-002): a randomised, controlled, phase 2 trial. Lancet Oncol 16:908-918

28. Robert C, Joshua AM, Weber JS, Ribas A, Hodi FS, Kefford RF et al. (2014) Pembrolizumab (Pembro; MK-3475) for advanced melanoma (MEL): randomized comparison of two dosing schedules. Ann Oncol 25 (suppl 4): doi: 10.1093/annonc/mdu438.42

29. Robert C, Schachter J, Long GV, Arance A, Grob JJ, Mortier L et al (2015) Pembrolizumab versus ipilimumab in advanced melanoma. N Engl J Med 372:2521-2532

30. Robert C, Ribas A, Wolchok JD, Hodi FS, Hamid O, Kefford R et al (2014) Anti-programmed-death-receptor-1 treatment with pembrolizumab in ipilimumab-refractory advanced melanoma: a randomised dose-comparison cohort of a phase 1 trial. Lancet 384:1109-1117

31. Herbst RS, Baas P, Kim DW, Felip E, Pérez-Gracia JL, Han JY et al (2015) Pembrolizumab versus docetaxel for previously treated, PDL1-positive, advanced non-small-cell lung cancer (KEYNOTE010): a randomised controlled trial. Lancet. doi:10.1016/S01406736(15)01281-7 